\title{
High-Performance Triangular Miniaturized-LEDs for High Current and Power Density Applications
}

Shunpeng Lu, *, Yiping Zhang," Zi-Hui Zhang, Binbin Zhu, Haiyang Zheng, Swee Tiam Tan,* and Hilmi Volkan Demir*

Cite This: ACS Photonics 2021, 8, 2304-2310

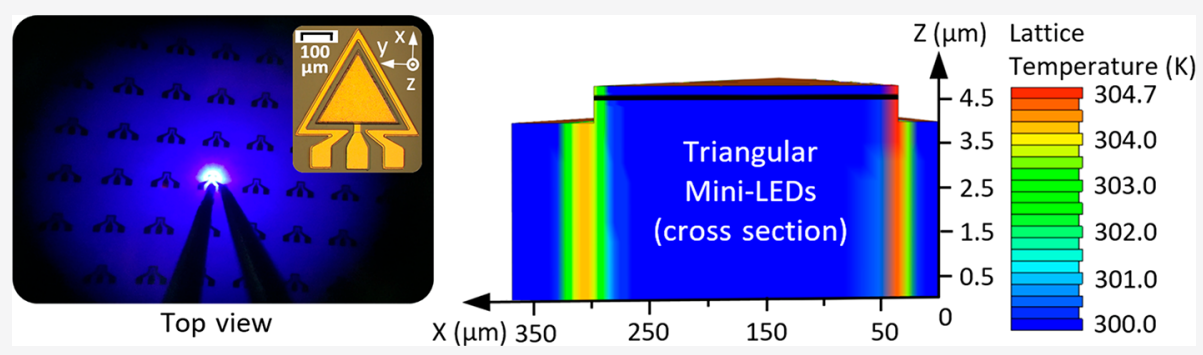

ABSTRACT: This work proposes an effective electrode length model and reveals for the first time the relationship between this model and the mesa shape effect. On the basis of this model, we demonstrate high-performance triangular miniaturized-LEDs (miniLEDs) and benchmark to the conventional square, and circular shapes of the same mesa area. Here, we systematically study the impact of shaping in mini-LEDs both theoretically and experimentally, which is fundamentally different than that of the conventional regular-sized LEDs. We find that, at the current level of $200 \mathrm{~mA}$, the triangular mini-LEDs deliver an enhancement of $36.4 \%$ in the optical output power and a decrease of $9.6 \%$ for the forward voltage compared to the commonly used square ones, and also an enhancement of $24.6 \%$ in the optical output power and a decrease of $14.3 \%$ for the forward voltage compared to the circular ones. The superior optical performance is proved to result from longer effective n-electrode length in the case of the triangular mini-LEDs, which suppresses the self-heating effect and thus well preserves the internal quantum efficiency, whereas the light extraction efficiency and the heat dissipation for the triangular shape are not significantly increased for such small mesa sizes, unlike conventional broad-area LEDs. Meanwhile, the reduced voltage is revealed to stem from the decreased n-GaN resistance. Different than conventional LEDs, these findings therefore indicate that the effective n-electrode length matters substantially for the miniaturized-LEDs.

KEYWORDS: micro-LEDs, self-heating, effective n-electrode length, backlit, shape effects, gallium nitride

I $\mathrm{n}$ recent years, small-sized LEDs in a flip-chip configuration have been intensively studied to explore various applications including visible light communication (VLC), displays, and biomedical treatment. ${ }^{1-5}$ For instance, mini-LEDs and microLEDs play an important role in making sunlight-readable emissive displays and enhancing the dynamic range of liquid crystal displays (LCDs). To achieve high dynamic-range miniLED backlit LCDs and high-quality sunlight-readable emissive displays, high peak brightness of small-sized LEDs is required. ${ }^{6-8}$ However, the efficiency and lifetime of such flipchipped LEDs are limited due to the self-heating, which is severe particularly due to current crowding at the mesa edges. ${ }^{9,10}$ Such self-heating also limits the highest current density that the chips can reach, and the response speed is thus substantially sacrificed. ${ }^{4}$ While the further development of VLC and displays continues, such problems generate a bottleneck for future possible uses and performance of these miniaturized-LEDs (mini-LEDs). To address these issues, vertically structured mini-LEDs will provide a better solution for the current-crowding effect. ${ }^{11}$ However, such vertically structured mini-LEDs require additional fabrication processes, among which the laser lift-off process has typically a low yield. The required complex fabrication process with such a low yield leads to higher fabrication costs. Therefore, as an alternative, it is essential and valuable to tackle the current-crowding problem of flip-chip structured mini-LEDs.

Previously, Dawson's group showed that LEDs could reduce the self-heating and possibly enhance the response speed with

Received: March 22, 2021

Published: July 21, 2021 

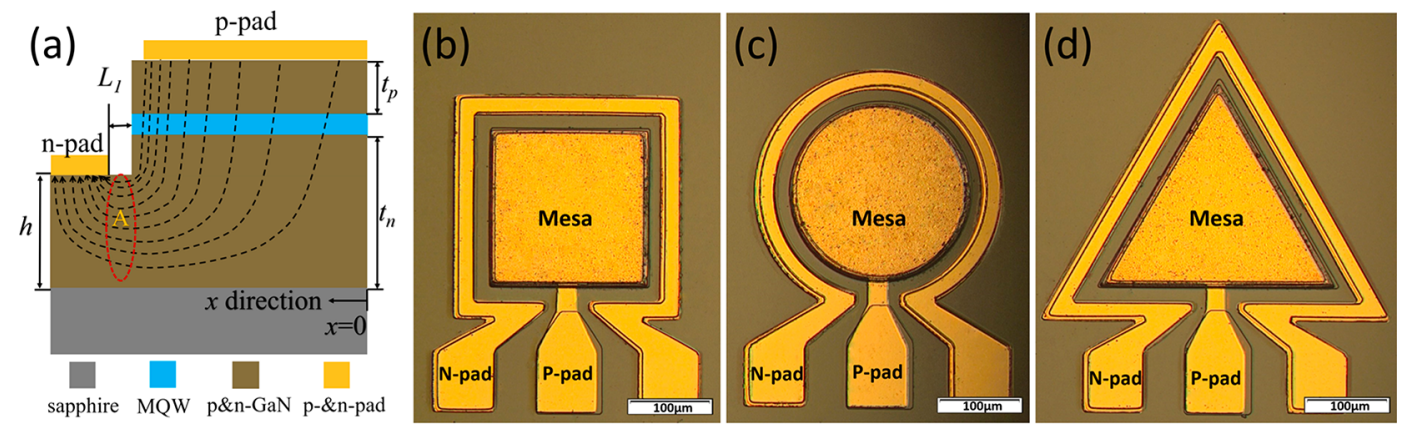

Figure 1. (a) Schematic cross-sectional illustration of the miniaturized LEDs showing the current paths inside the mini-LED from the p-pad to the $\mathrm{n}$-pad. Plan view of the fabricated mini-LEDs showing differently shaped mesas and contacts: microscopy photographs of (b) the square mini-LED, (c) the circular mini-LED, and (d) the triangular mini-LED, all with the same device mesa area.
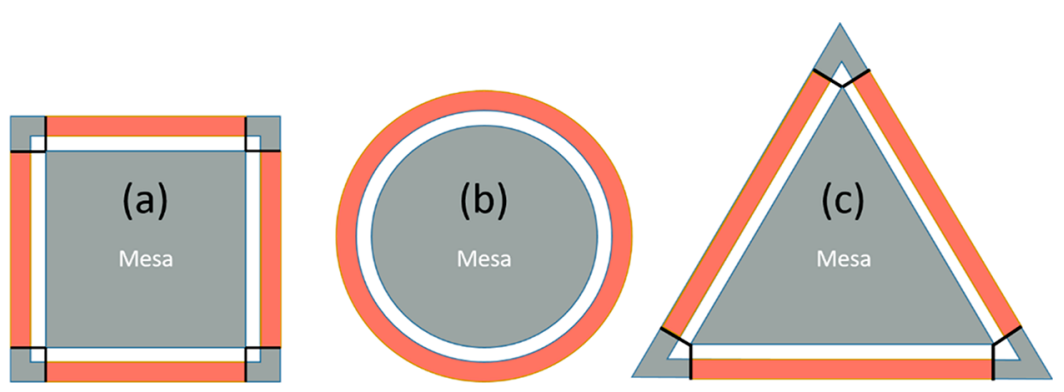

Figure 2. Schematic diagrams showing the effective n-electrode (the red part) for differently shaped mini-LEDs: (a) square, (b) circular, and (c) triangular.

shrinking size. ${ }^{4,12}$ Our group demonstrated low thermal-mass LEDs and also showed that smaller-sized LEDs deliver higher optical output power density and lower voltage bias due to the reduced self-heating. ${ }^{13}$ Nevertheless, with the size decreasing, on a certain display or across a certain size of lighting area, the total LED number increases and the vacant area without the active LED lighting will increase dramatically as the gap between neighboring LEDs limited by the fabrication cannot remain negligible. In other words, with the LED size decreasing and the gap area increasing, the total illuminance of a certain display or across a lighting area will be insufficient if the efficiency of the small-sized LEDs is kept unchanged. Therefore, enhancing the efficiency of small-sized LEDs with a certain mesa area is imperative to guarantee the required illuminance for the applications of VLC and displays under the background of chasing higher resolution. However, previous works focus on the size-dependent efficiency, ${ }^{14-19}$ whereas solutions to enhance the efficiency of such small-sized LEDs while keeping the same LED mesa area have not been studied in the case of mini-LEDs to date.

Also, Schubert et al. and Kang et al. previously reported that the self-heating could also be reduced by changing the electrode geometries. ${ }^{9,20}$ Through our theoretical calculation, we find that longer effective n-electrode length is beneficial for the higher efficiency, too. Here, the effective n-electrode length is the part of the n-electrode length that allows for effective current spreading from the p-electrode to the n-electrode. Figure 1a illustrates the current paths from the mesa area to the n-electrode and the current crowds at area A. As all the current from the mesa area goes to the n-electrode through area $\mathrm{A}$, the current density $\mathrm{j}$ in the area $\mathrm{A}$ is

$$
j=\frac{I}{h \times w}
$$

in which $I$ is the forward bias current, $h$ is the $n-G a N$ thickness under the n-electrode, and $w$ is the n-electrode length along the direction perpendicular to the cross section in Figure 1a. From eq 1 , we can see that, if $w$ is longer, the current density $j$ in the area A will be smaller. Therefore, for the LED with a certain mesa area, under the same current, the current crowding effect in the area $A$ will be alleviated when the nelectrode is longer. According to the Joule's law, at the same level of current, the total generated heat in the area $\mathrm{A}$ is

$$
J=I^{2} R t=\frac{I^{2} \rho t L_{1}}{h \times w}
$$

in which $\rho$ is the $\mathrm{n}-\mathrm{GaN}$ resistance, and $L_{1}$ is the length from the n-electrode edge to the mesa edge as shown in Figure 1a. From eq 2, at the same current level, when $w$ is longer, the total generated heat will be reduced. On the basis of eq 2, we can also deduce the relation between the local generated heat $J_{\text {loc }}$ and the current density $j$ :

$$
J_{\text {loc }}=j^{2} h w \rho t L_{1}
$$

From eq 3, we can observe that the local generated heat will increase dramatically with the square of the current density $j$. Therefore, with a shorter effective n-electrode length, at the same current density level, the current crowding effect at the mesa edge will be severer, the self-heating effect will be worse, and the local temperature will be further higher. What is more, for the small-sized LED design, the underlying physical mechanisms regarding the effective n-electrode length, including the local generated heat, $\mathrm{n}-\mathrm{GaN}$ resistance, and electron-hole recombination process, are of essential importance. However, in the previous works, these issues have not been investigated or addressed.

In Figure 2, we figure out the effective n-electrode length for different shapes all shown in red. The effective n-electrode 
refers to the n-electrode, which offers the shortest current path from the mesa area to the n-electrode area. Here, we use the middle line of the effective n-electrode (the red colored zone) in Figure 2 to calculate the effective n-electrode length. For the square shape, the effective $\mathrm{n}$-electrode length is $800.0 \mu \mathrm{m}$; for the circular one, it is $866.1 \mu \mathrm{m}$; and for the triangular one, it is $911.8 \mu \mathrm{m}$. On the basis of our calculations, we find that the triangular LEDs have the longest effective n-electrode length for different regular polygonal shapes. Therefore, given our effective n-electrode length model, our hypothesis is that the triangular LEDs enable the best performance owing to the reduced self-heating. To test our hypothesis and demonstrate the best performance of the triangular LEDs, we fabricated and studied systematically miniaturized-LEDs of triangular, circular, and typical square shapes carefully designed with the same mesa area, as shown in Figure $1 \mathrm{~b}-\mathrm{d}$. These devices all have different effective n-electrode lengths because of their different shapes. Among them, the triangular ones have the longest effective n-electrode length, whereas the square ones have the shortest effective n-electrode length.

Our experimental and numerical simulation results show that the triangular mini-LEDs deliver the highest output power and consume the lowest electrical power. Unlike conventional broad-area LEDs, these mini-LEDs, given their identical mesa size and depth, exhibit similar levels of light extraction efficiency, which is therefore not the reason why the triangular mini-LEDs outperform the others. The underlying reasons for high output power are the low lattice temperature at the mesa edges and high radiative recombination rate in the quantum wells. The $\mathrm{n}-\mathrm{GaN}$ resistances calculated for different shapes indicate that the lowest $\mathrm{n}-\mathrm{GaN}$ resistance contributes to the lowest power consumption for the triangular mini-LEDs among them all.

In this work, we grew and fabricated the aforementioned mini-LEDs. To do so, the LED epitaxy was grown on C-plane double polished sapphire substrates by our metal-organic chemical vapor deposition (MOCVD) system. More details on the growth can be found in our previous reports. ${ }^{13,21,22}$ The epi-wafers were subsequently processed into mini-LED dies shown in Figure $1 \mathrm{~b}-\mathrm{d}$. These mini-LEDs were designed in three shapes but all having an identical device mesa area: (1) circular mini-LEDs with a mesa diameter of $225.7 \mu \mathrm{m},(2)$ square mini-LEDs with a mesa side length of $200.0 \mu \mathrm{m}$, and (3) equilateral triangular mini-LEDs with a side length of 303.9 $\mu \mathrm{m}$. For these three sets of mini-LEDs, the mesa depth is 0.8 $\mu \mathrm{m}$, which is much shallower than the thickness of sapphire $(80 \mu \mathrm{m})$ even after dicing, as reported by Kim et al. ${ }^{23}$

Here, standard micro-fabrication techniques were used to fabricate the LED wafers in the flip-chip configuration. Mesa shapes of circle, square, and triangle were prepared by using reactive ion etching (RIE). The sidewalls were protected by a layer of $\mathrm{SiO}_{2}$, which also acts as a passivation layer to reduce the nonradiative recombination and current leakage. $\mathrm{Ni} / \mathrm{Ag}$ based metal layers, annealed in oxygen ambient after being deposited, were used as the reflective mirror. Ti/Au metal layers $(30 \mathrm{~nm} / 1000 \mathrm{~nm}$ thick) were deposited as the $\mathrm{p}$ - and $\mathrm{n}$ contact pads. The mesa shapes were surrounded by $\mathrm{n}$-contact pads $15 \mu \mathrm{m}$ away from the mesa edges. An LED tester (M2442S-9A Quatek Group) was used to measure the current-voltage characteristics for the fabricated LED chips, and the optical output power was measured by an Ocean Optics spectrometer (QE65000) attached to an integrating sphere. The temperature of these differently shaped mini-LEDs was measured by an IR camera (FAST-IR Telops Inc.).

The Monte Carlo method was used to study the light extraction efficiency (LEE) of these mini-LEDs in different shapes. $^{24,25}$ In the simulations, all surfaces of the LEDs were modeled to be of optical quality. The Snell's law was applied when the light travels through the structures and the interfaces. We assume that the angular distribution of the light emitted from the multiple quantum wells (MQWs) is uniform. The material thicknesses and refractive indices were taken as in Table 1, and the optical absorption level for the MQWs and p-

Table 1. Material Thicknesses and Refractive Indices at the Emission Wavelength of $450 \mathrm{~nm}$ Used for Calculating the LEE

\begin{tabular}{lcc}
\multicolumn{1}{c}{ material } & thickness $(\mu \mathrm{m})$ & refractive index \\
double polished sapphire substrate & 420 & 1.77 \\
$\mathrm{u}-\mathrm{GaN}$ & 3.00 & 2.40 \\
$\mathrm{n}-\mathrm{GaN}$ & 5.50 & 2.43 \\
$\mathrm{MQWs}$ & 0.08 & 2.54 \\
$\mathrm{Al}_{0.15} \mathrm{Ga}_{0.85} \mathrm{~N}$ & 0.02 & 2.42 \\
$\mathrm{p}-\mathrm{GaN}$ & 0.20 & 2.45 \\
\hline
\end{tabular}

$\mathrm{GaN}$ was set as $2000 \mathrm{~cm}^{-1}$ for calculating the LEE at the emission wavelength of $450 \mathrm{~nm}^{26}$ The optical flux emitted from the MQW was set at $1 \mathrm{~W} / 1000000$ rays for this simulation. ${ }^{27}$ To further understand the shape effect on the electrical and optical properties, additional three-dimensional (3D) numerical device modeling was performed by using APSYS. In these simulations, the LED device structures were set the same as our mini-LEDs shown in Figure $1 b-d$. More details on our numerical simulation parameters can be found in our previously published reports. ${ }^{13,21}$

Table 2 shows the LEE computed for the square, triangular, and circular mini-LEDs based on the Monte Carlo method.

Table 2. LEE of the Square, Triangular, and Circular MiniLEDs Based on the Monte Carlo Method

\begin{tabular}{cccc} 
& square & triangular & circular \\
LEE $(\%)$ & 16.85 & 16.84 & 16.83 \\
\hline
\end{tabular}

From Table 2, it is clearly seen that the LEE of the square mini-LEDs is slightly higher than that of the triangular and circular mini-LEDs. These results indicate that the LEE is nearly the same for these mini-LEDs with different shapes and there is no major variation between them, unlike conventional broad-area LEDs in which the mesa shape dramatically affects the LEE. This is because the sidewall depth of our mini-LEDs is only $800 \mathrm{~nm}$. Also according to a previous report, ${ }^{23}$ the impact of $800 \mathrm{~nm}$ deep sidewalls on the LEE can be negligible compared to the impact of $80 \mu \mathrm{m}$ sidewalls on the LEE.

On the other hand, the optical and electrical performances of these mini-LEDs experimentally exhibit great dependence on shapes. Figure 3 shows the output power for the square, circular, and triangular mini-LEDs. Figure $3 \mathrm{a}$ is the experimental results, and Figure $3 b$ is the $3 \mathrm{D}$ numerically computed results. From Figure 3, one can clearly see that the simulated results match reasonably well with the experimental results, except for the slight roll-off toward high current. Both experimentally and numerically, triangular mini-LEDs deliver the highest output power, the circular ones rank the second, 

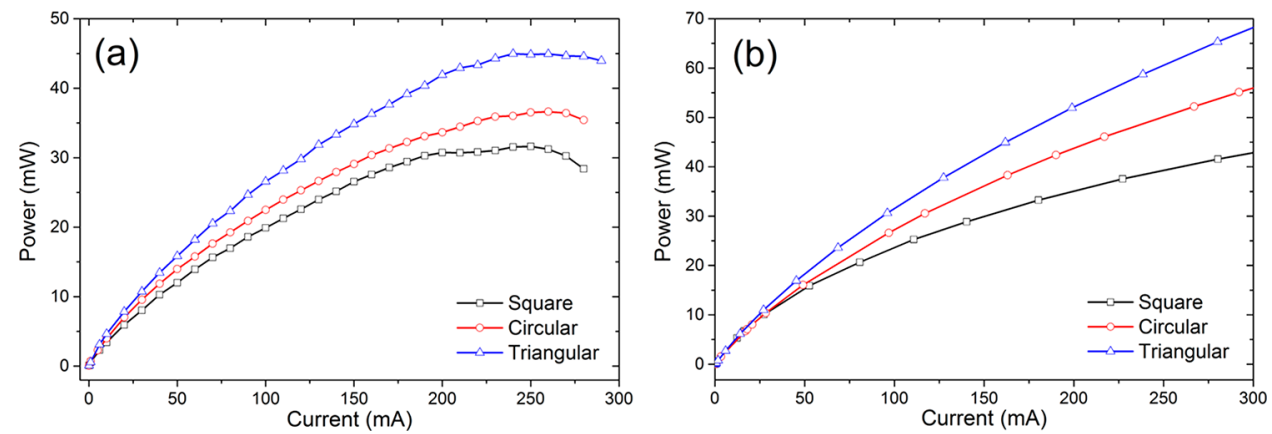

Figure 3. Output power of the square, circular, and triangular mini-LEDs: (a) experimental and (b) three-dimensional numerically computed results.
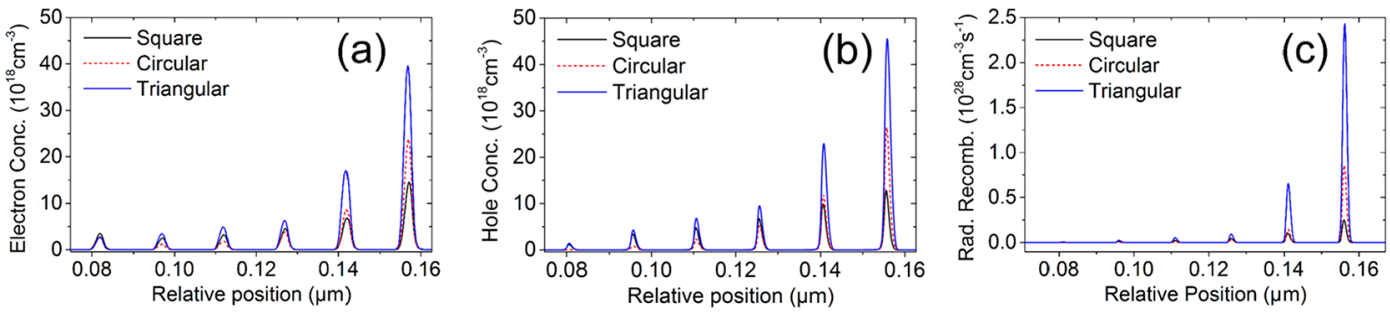

Figure 4. Three-dimensional numerically computed results of (a) electron concentration, (b) hole concentration, and (c) radiative recombination rates of the square, circular, and triangular mini-LEDs.

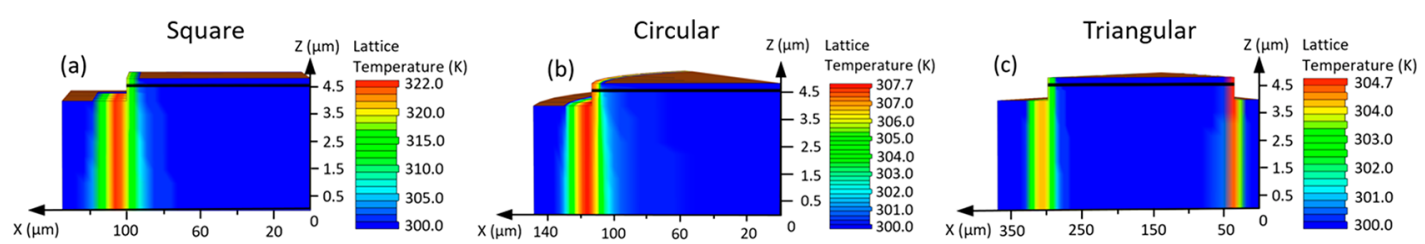

Figure 5. Lattice temperature distribution of three-dimensional simulation results for (a) the square (1/4 part cut by two axes of symmetry), (b) the circular (sector), and (c) the triangular ( $1 / 2$ part cut by one axis of symmetry) mini-LEDs.
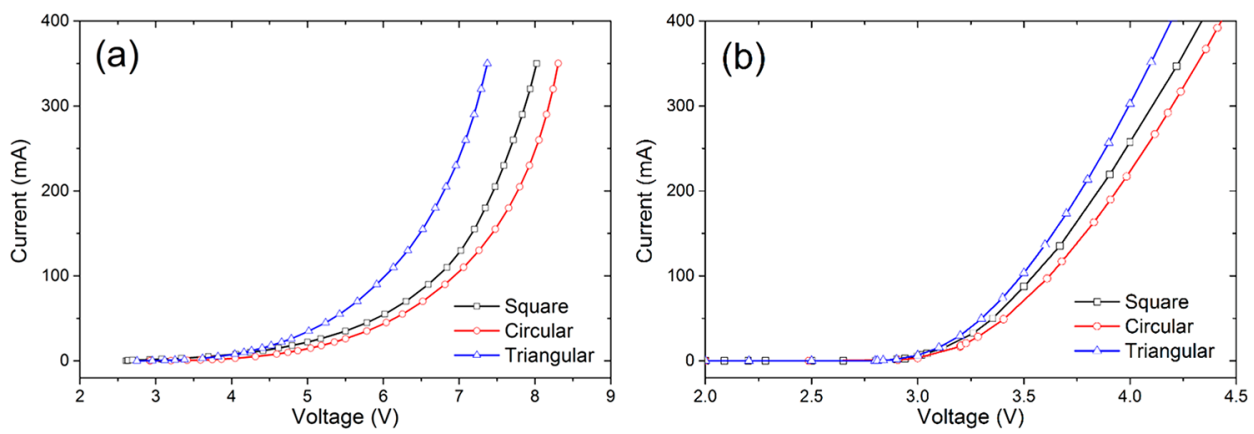

Figure 6. (a) Experimental and (b) three-dimensional numerical simulation results of $I-V$ characteristics for differently shaped mini-LEDs.

and the square ones show the lowest. This is because they have different effective n-electrode lengths. On the basis of the aforementioned calculations, the effective n-electrode length of the square shaped mini-LEDs is $800.0 \mu \mathrm{m}$, that of the circular one is $866.1 \mu \mathrm{m}$, and that of the triangular one is $911.8 \mu \mathrm{m}$. Longer effective n-electrode length is beneficial for the current spreading and a uniform current density distribution will alleviate the local heating, which makes a higher internal quantum efficiency (IQE) and output power possible. This matches well with our theoretical calculation results that longer effective n-electrode length leads to increased output power.

To understand the underlying physical mechanisms, 3D numerical simulations were performed to study the electron concentration and hole concentration, and radiative recombination rate in the quantum wells. Figure $4 a-c$ shows the electron concentration, hole concentration, and the radiative recombination rate of the $3 \mathrm{D}$ simulations, respectively. From Figure $4 a-c$, we can see that the electron concentration, the hole concentration, and the radiative recombination rate are dominated by the last two quantum wells. In the last two quantum wells, triangular mini-LEDs present the highest electron and hole concentrations, whereas the square ones show the lowest. This leads to the highest radiative recombination rate for the triangular mini-LEDs and the lowest for the square ones. Therefore, the triangular mini- 
(a)

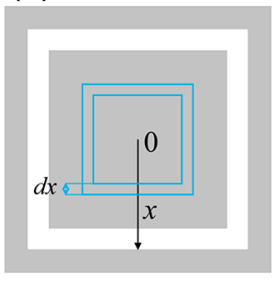

(b)

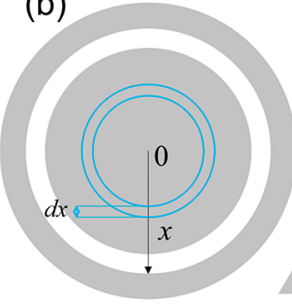

(c)

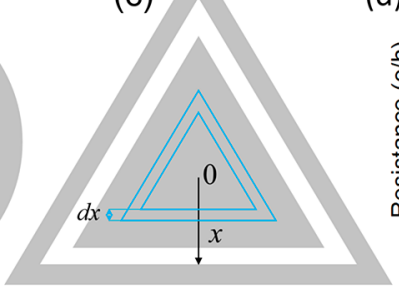

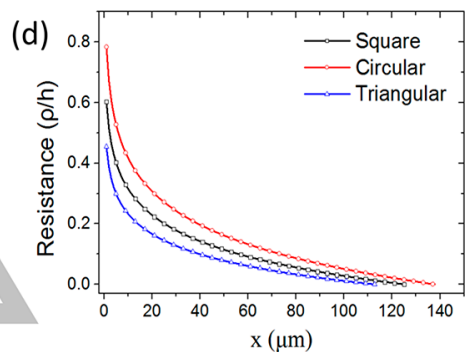

Figure 7. Schematic diagrams showing the $x$ direction and an infinitely small increment $\mathrm{d} x$ for differently shaped mini-LEDs: (a) square, (b) circular, and (c) triangular. (d) The resistance calculated as a function of $x$ for different shapes.

LEDs deliver the highest output power and the square ones deliver the lowest.

To further understand the effect of the effective electrode length on the generated heat, we studied the lattice temperature of different shapes. Figure 5 shows the 3D simulation results of the lattice temperature of the three sets of differently shaped mini-LEDs. From Figure 5, we can observe that, compared to $322.0 \mathrm{~K}$ of the square mini-LEDs and 307.7 $\mathrm{K}$ of the circular ones, the triangular ones show the lowest, $304.7 \mathrm{~K}$, of the lattice temperatures at the mesa edges. Furthermore, we also experimentally measured the temperature of these mini-LEDs using an IR camera. The measurement was carried out under the forward current of $30 \mathrm{~mA}$. The experimental results show that the temperatures of our triangular, circular, and square mini-LEDs increase by 1.35 , 2.65 , and $4.96{ }^{\circ} \mathrm{C}$, respectively. This measurement clearly proves that the self-heating effect is relieved the most in the triangular mini-LEDs.

Figure 6 shows the current-voltage characteristics for our differently shaped mini-LEDs. From Figure 6, we can observe that the experimental results match well with the $3 \mathrm{D}$ simulation results. It is clear that, at the same current level, the triangular mini-LEDs feature the lowest bias voltage, and the circular ones possess the highest bias voltage. To better understand the observed phenomenon in the $I-V$ characteristics for the investigated mini-LEDs, we adopt the current paths for the mini-LEDs as depicted in Figure 1a. In this illustration, we can see that the current from the p-pad finally flows to the n-pad through the $\mathrm{p}-\mathrm{GaN}$ region, the MQW region, and the $\mathrm{n}-\mathrm{GaN}$ region. Since all the studied devices have the identical mesa area and epitaxial thickness for the pGaN layer and the MQW region, the resistances caused by these two parts are the same for all the devices. Meanwhile, all the mini-LEDs have the same mesa depth, which causes an identical level of resistance for the $\mathrm{n}-\mathrm{GaN}$ region with a thickness of $\left(t_{n}-h\right)$. However, after reaching the rest of the nGaN layer with the thickness of $h$, the current will further need to travel laterally before the arrival at the n-pad. Since the resistance $R$ in the $\mathrm{n}-\mathrm{GaN}$ layer is critical in determining the voltage consumption for different mini-LEDs, it is essential to calculate the value of $\mathrm{n}-\mathrm{GaN}$ resistance for different shapes.

Here, we define the $\mathrm{n}-\mathrm{GaN}$ resistance $R=\rho l / S$, in which $\rho$ is the $\mathrm{n}-\mathrm{GaN}$ resistivity, $l$ is the current path from p-pad to $\mathrm{n}$-pad middle line, and $S$ is the cross-sectional area of the current flow. All the three shaped mini-LEDs have the same $n-G a N$ resistivity $\rho$. Meanwhile, we also know that $S=h \times p$, in which $h$ is the $\mathrm{n}-\mathrm{GaN}$ thickness as shown in Figure 1a, and $p$ is the perimeter for different shapes. We set the center of the different shapes as 0 , and $x$ is the distance from the center perpendicular to the $\mathrm{n}$-pad middle line for all the three different shapes as illustrated in Figure 7. As the perimeter $p$ is $x$ dependent, we need to calculate the $\mathrm{n}-\mathrm{GaN}$ resistance using integration. Take the square shape as an example. For any $x$ on the mesa area, when $x$ changes by an infinitely small increment $\mathrm{d} x$, the differential resistance due to this $\mathrm{d} x$ length should be $\mathrm{d} R_{\mathrm{s}}=\frac{\rho}{8 x h} \mathrm{~d} x$, where $\mathrm{d} x$ is $l, 8 x$ is the perimeter, and $h$ is the $\mathrm{n}$ GaN thickness. Similarly, for the circular shape, $\mathrm{d} R_{\mathrm{c}}=\frac{\rho}{2 \pi x h} \mathrm{~d} x$, and for the triangular shape, $\mathrm{d} R_{\mathrm{t}}=\frac{\rho}{6 \sqrt{3} x h} \mathrm{~d} x$. For the square mini-LEDs, the distance from the square center perpendicular to the $\mathrm{n}$-pad middle line is $125.00 \mu \mathrm{m}$; for the triangular miniLEDs, the distance from the triangular center perpendicular to the $\mathrm{n}$-pad middle line is $112.74 \mu \mathrm{m}$; and for the circular miniLEDs, the distance from the circular center perpendicular to the n-pad middle line is $137.85 \mu \mathrm{m}$. Thus, the $\mathrm{n}-\mathrm{GaN}$ resistances from any position $x$ to the n-electrode are

$$
\begin{aligned}
R_{\mathrm{s}}(x) & =\int_{x}^{125.00} \frac{\rho}{8 x h} \mathrm{~d} x=(0.604-\ln x / 8) \rho / h \\
(0 & <x<125.00) \\
R_{\mathrm{c}}(x) & =\int_{x}^{137.85} \frac{\rho}{2 \pi x h} \mathrm{~d} x=(0.787-\ln x / 2 \pi) \rho / h \\
(0 & <x<137.85) \\
R_{\mathrm{t}}(x) & =\int_{x}^{112.74} \frac{\rho}{6 \sqrt{3} x h} \mathrm{~d} x=(0.455-\ln x / 6 \sqrt{3}) \rho / h \\
(0 & <x<112.74)
\end{aligned}
$$

for the square, the circular, and the triangular mini-LEDs, respectively. On the basis of eqs 4-6, we further calculated the $\mathrm{n}$-GaN resistance as a function of $x$ for different shapes, as shown in Figure $7 d$. From Figure $7 d$, we can obtain that the triangular mini-LEDs have the smallest $\mathrm{n}-\mathrm{GaN}$ resistance $\left(R_{\mathrm{t}}\right)$ and the circular ones have the largest $\mathrm{n}-\mathrm{GaN}$ resistance $\left(R_{\mathrm{c}}\right)$ for a certain $x$ position. As the voltage drop across the $n-G a N$ resistance is the current multiplied by the $\mathrm{n}-\mathrm{GaN}$ resistance, the $\mathrm{n}-\mathrm{GaN}$ voltage drop for the triangular mini-LEDs is the smallest and that for the circular ones is the largest. Consequently, the triangular mini-LEDs exhibit the smallest forward voltage bias and the circular ones, the largest, at the same current level. These results explain the bias performance of these mini-LEDs as shown in Figure 6.

To demonstrate whether our model works well on miniLEDs with smaller sizes, we further fabricated differently shaped mini-LEDs with a mesa side length of $50 \mu \mathrm{m}$ for the square and triangular mini-LEDs and a diameter of $50 \mu \mathrm{m}$ for the circular mini-LEDs. On the basis of our calculation, the effective n-electrode length of the triangular mini-LEDs is 1.14 

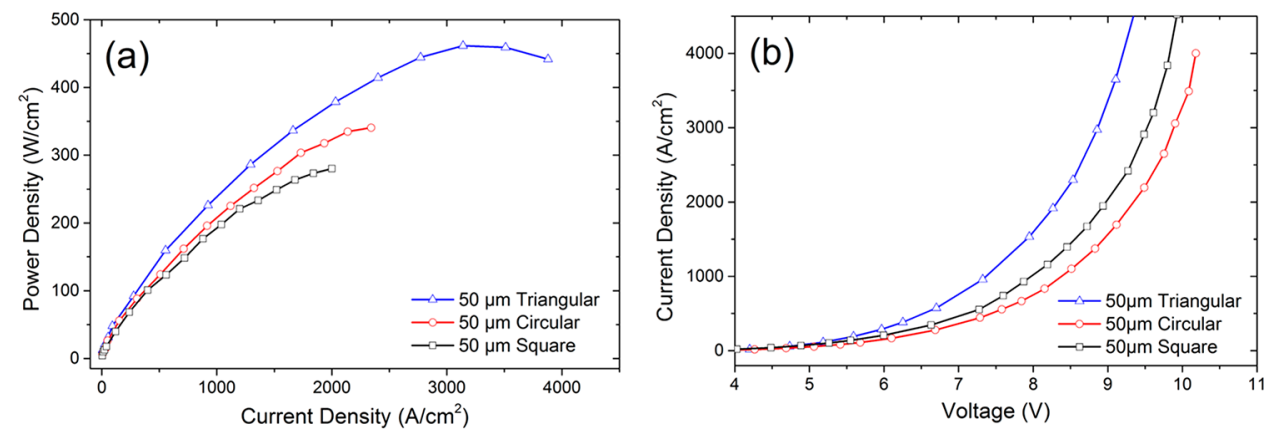

Figure 8. Experimental results of (a) output power and (b) $I-V$ characteristics for differently shaped mini-LEDs.

times longer than that of the typical square ones for any size with the same mesa area. For the circular mini-LEDs, we set the distance from the n-electrode edge to the mesa edge to be $0.2 r$ (where $r$ is the mesa radius of the circular mini-LEDs). The effective n-electrode length ratio for the square, circular, and triangular mini-LEDs with the same mesa area based on our model is 1.00:1.06:1.14. In summary, for any size range of the mini-LEDs, the triangular ones have the longest effective nelectrode length among all of the square, circular, and triangular with the same mesa area. Therefore, $50 \mu \mathrm{m}$ triangular mini-LEDs lead to the best output power density, whereas the square ones exhibit the lowest output power density, which matches well with our experimental demonstration as presented in Figure 8a. Similarly, on the basis of our $\mathrm{n}$-GaN resistance model, the $50 \mu \mathrm{m}$ triangular mini-LEDs show the highest current density and the circular ones, the lowest current density, which agrees well with our experimental results as shown in Figure $8 \mathrm{~b}$.

In conclusion, we proposed and demonstrated the effective n-electrode length model and showed differently shaped miniLEDs with the same active lighting area. Surprisingly, we found that the triangular mini-LEDs deliver more output optical power than the commonly used square ones (36.4\% more) and the circular ones $(24.6 \%$ more) at a fixed current level (200 $\mathrm{mA}$ ) for the same mesa size. Furthermore, unexpectedly, we uncovered that the light extraction efficiencies and the heat dissipation of all these mini-LEDs of different shapes are similar, which cannot then explain the substantial enhancement observed in the output power of the triangular mini-LEDs compared to the square and circular counterparts. Here, we found that the triangular mini-LEDs exhibit superior performance because of their geometry that leads to the longest effective n-electrode length and the lowest $\mathrm{n}-\mathrm{GaN}$ resistance. Our numerical 3D simulations show that the triangular miniLEDs possess the highest output power due to the highest radiative recombination rate and the lowest lattice temperature as a result of the lowest $\mathrm{n}-\mathrm{GaN}$ resistance. Calculating the n$\mathrm{GaN}$ resistance, we find that the triangular mini-LEDs consume the lowest electrical power due to the smallest $n$ $\mathrm{GaN}$ resistance. Thus, the longer effective n-electrode length is a critically important factor that should be considered for miniLEDs to achieve high output power.

\section{AUTHOR INFORMATION}

\section{Corresponding Authors}

Shunpeng Lu - Luminous! Centre of Excellence for Semiconductor Lighting and Displays, School of Electrical and Electronic Engineering, Nanyang Technological
University, Singapore 639798, Singapore; 이이이.org/ 0000-0002-6289-3663; Email: lush0007@ntu.edu.sg

Swee Tiam Tan - Luminous! Centre of Excellence for Semiconductor Lighting and Displays, School of Electrical and Electronic Engineering, Nanyang Technological University, Singapore 639798, Singapore; School of Energy and Chemical Engineering, Xiamen University Malaysia, 43900 Sepang, Selangor, Malaysia; Email: sweetiam.tan@ xmu.edu.my

Hilmi Volkan Demir - Luminous! Centre of Excellence for Semiconductor Lighting and Displays, School of Electrical and Electronic Engineering, Nanyang Technological University, Singapore 639798, Singapore; School of Physics and Mathematical Sciences, Nanyang Technological University, Singapore 637371, Singapore; Department of Electrical and Electronics, Department of Physics, and UNAM-Institute of Material Science and Nanotechnology, Bilkent University, Ankara TR-06800, Turkey; (1) orcid.org/ 0000-0003-1793-112X; Email: volkan@ stanfordalumni.org

\section{Authors}

Yiping Zhang - Luminous! Centre of Excellence for Semiconductor Lighting and Displays, School of Electrical and Electronic Engineering, Nanyang Technological University, Singapore 639798, Singapore

Zi-Hui Zhang - Luminous! Centre of Excellence for Semiconductor Lighting and Displays, School of Electrical and Electronic Engineering, Nanyang Technological University, Singapore 639798, Singapore; Key Laboratory of Electronic Materials and Devices of Tianjin, School of Electronics and Information Engineering, Hebei University of Technology, Tianjin 300401, People's Republic of China; () orcid.org/0000-0003-0638-1118

Binbin Zhu - Luminous! Centre of Excellence for Semiconductor Lighting and Displays, School of Electrical and Electronic Engineering, Nanyang Technological University, Singapore 639798, Singapore

Haiyang Zheng - Luminous! Centre of Excellence for Semiconductor Lighting and Displays, School of Electrical and Electronic Engineering, Nanyang Technological University, Singapore 639798, Singapore

Complete contact information is available at: https://pubs.acs.org/10.1021/acsphotonics.1c00430

\section{Author Contributions}

${ }^{\#}$ S.L. and Y.Z. contributed in equal measure to this work.

Notes

The authors declare no competing financial interest. 


\section{ACKNOWLEDGMENTS}

This work is supported by the Singapore National Research Foundation under Grant No. NRF-CRP-6-2010-2 and the Singapore Agency for Science, Technology and Research (A*STAR) SERC Pharos Program under Grant No. 1527300025. H.V.D also gratefully acknowledges TUBA.

\section{REFERENCES}

(1) Day, J.; Li, J.; Lie, D. Y. C.; Bradford, C.; Lin, J. Y.; Jiang, H. X. III-Nitride full-scale high-resolution microdisplays. Appl. Phys. Lett. 2011, 99 (3), 031116.

(2) Gu, E.; Jeon, C. W.; Choi, H. W.; Rice, G.; Dawson, M. D.; Illy, E. K.; Knowles, M. R. H. Micromachining and dicing of sapphire, gallium nitride and micro LED devices with UV copper vapour laser. Thin Solid Films 2004, 453-454, 462-466.

(3) Jovicic, A.; Li, J.; Richardson, T. Visible light communication: opportunities, challenges and the path to market. IEEE Commun. Mag. 2013, 51 (12), 26-32.

(4) McKendry, J. J. D.; Massoubre, D.; Zhang, S.; Rae, B. R.; Green, R. P.; Gu, E.; Henderson, R. K.; Kelly, A. E.; Dawson, M. D. VisibleLight Communications Using a CMOS-Controlled Micro-LightEmitting-Diode Array. J. Lightwave Technol. 2012, 30 (1), 61-67.

(5) Yeh, N. G.; Wu, C.-H.; Cheng, T. C. Light-emitting diodesTheir potential in biomedical applications. Renewable Sustainable Energy Rev. 2010, 14 (8), 2161-2166.

(6) Hsiang, E.-L.; Yang, Q.; He, Z.; Zou, J.; Wu, S.-T. Halo effect in high-dynamic-range mini-LED backlit LCDs. Opt. Express 2020, 28 (24), 36822-36837.

(7) Tan, G.; Huang, Y.; Li, M.-C.; Lee, S.-L.; Wu, S.-T. High dynamic range liquid crystal displays with a mini-LED backlight. Opt. Express 2018, 26 (13), 16572-16584.

(8) Huang, Y.; Hsiang, E.-L.; Deng, M.-Y.; Wu, S.-T. Mini-LED, Micro-LED and OLED displays: present status and future perspectives. Light: Sci. Appl. 2020, 9 (1), 105.

(9) Guo, X.; Schubert, E. F. Current crowding and optical saturation effects in GaInN/GaN light-emitting diodes grown on insulating substrates. Appl. Phys. Lett. 2001, 78 (21), 3337-3339.

(10) Du, C.; Jing, L.; Jiang, C.; Liu, T.; Pu, X.; Sun, J.; Li, D.; Hu, W. An effective approach to alleviating the thermal effect in microstripe array-LEDs via the piezo-phototronic effect. Mater. Horiz. 2018, 5 (1), 116-122.

(11) Tian, P.; McKendry, J. J. D.; Gu, E.; Chen, Z.; Sun, Y.; Zhang, G.; Dawson, M. D.; Liu, R. Fabrication, characterization and applications of flexible vertical InGaN micro-light emitting diode arrays. Opt. Express 2016, 24 (1), 699-707.

(12) Gong, Z.; Jin, S.; Chen, Y.; McKendry, J.; Massoubre, D.; Watson, I. M.; Gu, E.; Dawson, M. D. Size-dependent light output, spectral shift, and self-heating of $400 \mathrm{~nm}$ InGaN light-emitting diodes. J. Appl. Phys. 2010, 107 (1), 013103.

(13) Lu, S.; Liu, W.; Zhang, Z.-H.; Tan, S. T.; Ju, Z.; Ji, Y.; Zhang, X.; Zhang, Y.; Zhu, B.; Kyaw, Z.; Hasanov, N.; Sun, X. W.; Demir, H. V. Low thermal-mass LEDs: size effect and limits. Opt. Express 2014, 22 (26), 32200-32207.

(14) Konoplev, S. S.; Bulashevich, K. A.; Karpov, S. Y. From LargeSize to Micro-LEDs: Scaling Trends Revealed by Modeling. Phys. Status Solidi A 2018, 215 (10), 1700508.

(15) Olivier, F.; Tirano, S.; Dupré, L.; Aventurier, B.; Largeron, C.; Templier, F. Influence of size-reduction on the performances of GaNbased micro-LEDs for display application. J. Lumin. 2017, 191, 112116.

(16) Singh, S.; Kumar, S.; Pal, S.; Dhanavantri, C. Performances of p-side down vertical InGaN/GaN blue light-emitting diodes with chip size. Opt. Laser Technol. 2017, 95, 165-171.

(17) Tian, P.; McKendry, J. J. D.; Gong, Z.; Guilhabert, B.; Watson, I. M.; Gu, E.; Chen, Z.; Zhang, G.; Dawson, M. D. Size-dependent efficiency and efficiency droop of blue InGaN micro-light emitting diodes. Appl. Phys. Lett. 2012, 101 (23), 231110.
(18) Wong, M. S.; Lee, C.; Myers, D. J.; Hwang, D.; Kearns, J. A.; Li, T.; Speck, J. S.; Nakamura, S.; DenBaars, S. P. Size-independent peak efficiency of III-nitride micro-light-emitting-diodes using chemical treatment and sidewall passivation. Appl. Phys. Express 2019, 12 (9), 097004.

(19) Zhu, J.; Takahashi, T.; Ohori, D.; Endo, K.; Samukawa, S.; Shimizu, M.; Wang, X.-L. Near-Complete Elimination of SizeDependent Efficiency Decrease in GaN Micro-Light-Emitting Diodes. Phys. Status Solidi A 2019, 216, 1900380.

(20) Park, Y. S.; Lee, H. G.; Yang, C.-M.; Kim, D.-S.; Bae, J.-H.; Cho, S.; Lee, J.-H.; Kang, I. M. Fabrication and Characterization of a GaN Light-emitting Diode (LED) with a Centered Island Cathode. J. Opt. Soc. Korea 2012, 16, 349-353.

(21) Zhang, Z.-H.; Tan, S. T.; Ju, Z.; Liu, W.; Ji, Y.; Kyaw, Z.; Dikme, Y.; Sun, X.; Demir, H. V. On the Effect of Step-Doped Quantum Barriers in InGaN/GaN Light Emitting Diodes. J. Disp. Technol. 2013, 9 (4), 226-233.

(22) Ju, Z. G.; Liu, W.; Zhang, Z. H.; Tan, S. T.; Ji, Y.; Kyaw, Z. B.; Zhang, X. L.; Lu, S. P.; Zhang, Y. P.; Zhu, B. B.; Hasanov, N.; Sun, X. W.; Demir, H. V. Improved hole distribution in InGaN/GaN lightemitting diodes with graded thickness quantum barriers. Appl. Phys. Lett. 2013, 102 (24), 243504.

(23) Kim, J. Y.; Kwon, M. K.; Kim, J. P.; Park, S. J. Enhanced light extraction from triangular GaN-Based light-emitting diodes. IEEE Photonics Technol. Lett. 2007, 19 (21-24), 1865-1867.

(24) Lee, S. J. Analysis of light-emitting diodes by Monte Carlo photon simulation. Appl. Opt. 2001, 40 (9), 1427-1437.

(25) Badano, A.; Kanicki, J. Monte Carlo analysis of the spectral photon emission and extraction efficiency of organic light-emitting devices. J. Appl. Phys. 2001, 90 (4), 1827-1830.

(26) Chung, S. C.; Ho, P. C.; Li, D. R.; Lee, T. X.; Yang, T. H.; Sun, C. C. Effect of chip spacing on light extraction for light-emitting diode array. Opt. Express 2015, 23 (11), A640-9.

(27) Wang, X. H.; Lai, P. T.; Choi, H. W. The contribution of sidewall light extraction to efficiencies of polygonal light-emitting diodes shaped with laser micromachining. J. Appl. Phys. 2010, 108 (2), 023110. 